PII: S0735-1933(02)00349-4

\title{
RESPONSE OF A TWO PHASE SYSTEM SUBJECT TO OSCILLATIONS INDUCED BY THE MOTION OF ITS SUPPORT STRUCTURE
}

\author{
J. S. Jayakumar, R. B. Grover \\ Bhabha Atomic Research Centre, \\ Mumbai, India. 400085 \\ and V.H. Arakeri, \\ Indian Institute of Science, \\ Bangalore. India. 560012
}

(Communicated by A.R. Balakrishnan)

\begin{abstract}
In many parts of the world, seawater desalination offers one of the most promising alternatives for supplying the required potable water. The desalination is an energy intensive process and use of nuclear reactors as the energy source for desalination is an attractive option. To ensure reactor safety for a barge-mounted plant, multiple systems for removing heat from the core even after the nuclear reactor is shutdown, need to be provided. One of the Residual Heat Removal Systems (RHRS) has to be energy independent and hence has to be based on the principles of natural circulation. A three-loop energy independent RHRS, in which the second loop is working in two-phase, is described. The barge will be invariably subjected to oscillations due to seawater movements. The RHRS should be able to perform its intended functions even when the barge is oscillating. If the natural frequency of oscillations of the RHRS is close to the frequency of oscillations of the barge, resonance may occur and lead to deterioration of heat removal capability. Hence, it is necessary to determine the natural frequency of oscillation of such a system. Temperature and quality distribution of the working fluid required for the estimation of natural frequency of the system is calculated by solving momentum balance and energy balance equations for the loops of the RHRS. Various terms of the governing equation describing oscillatory behaviour of non-isothermal, twophase closed loop system are derived. The overall equation is solved using fourth order Runge-Kutta method. The response of the system for various initial conditions is computed. ๑ 2002 Elsevier Science Ltd
\end{abstract}

\section{Introduction}

It has been estimated that on a global basis, about $12 \%$ of the world population lacks safe water supplies and 56\% has no adequate sanitation. Matching the ever-growing demands with the limited 
supply of finite resources has led to tremendous stress on natural fresh water. In many parts of the world, seawater desalination offers one of the most promising alternatives for supplying the required potable water.

The desalination is an energy intensive process and use of nuclear reactors as the energy source for desalination is an attractive option. A barge-mounted nuclear reactor desalination plant can provide drinking water in many coastal regions of our country. A number of Nuclear Desalination plants are operating worldwide and one land based plant is being built at Kalpakkam in India. For barge mounted desalination plants, various reactor system concepts have been examined. Pressurised Water Reactors of small rating are found to be the best choice for a barge-mounted reactor, which is coupled to a desalination plant [1]. A barge mounted desalination plant is likely to be an islanded system. Therefore, to ensure reactor safety [2], it is necessary to provide passive systems for removing residual heat from the core, which is generated after the nuclear reactor is shutdown. A possible Residual Heat Removal System (RHRS) based on three loops in natural circulation; in which one of the loops is working in two-phase is shown in Fig. 1 [3]. The first loop of the RHRS circulates reactor coolant between the core and the heat exchanger, which is located at a higher elevation than the core. In the second loop, the working fluid is converted to steam in the heat exchanger and rises up. The steam is condensed in a condenser, which is at

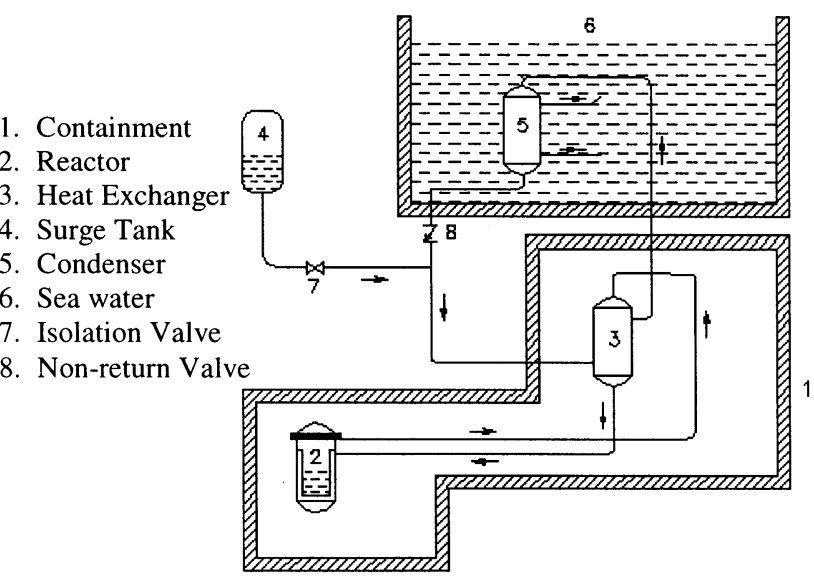

FIG.1

Schematic of Natural Circulation Residual Heat Removal System

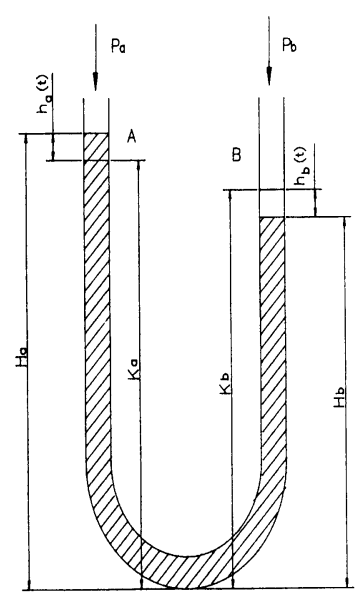

FIG. 2

Non-isothermal Open Loop System 
a higher elevation and the condensate flows back to the heat exchanger. The third loop is a seawater loop. The cold seawater enters the shell side through the lower nozzle of the condenser, takes away heat from the condensing steam and flows out through the upper nozzle. The flow of seawater in the condenser is also by natural circulation. There are three regimes of heat transfer viz., pre-heating zone, boiling zone and superheating zone, in the heat exchanger. Condensing and Subcooling zones are present in the Condenser.

A barge will be subjected to various oscillations due to seawater movements. The RHRS should be able to perform its intended functions even when the barge is oscillating. It is necessary to find out the natural frequency of oscillation of such a system. If the natural frequency of oscillations of the RHRS is close to the frequency of oscillations of the barge, resonance may occur and lead to deterioration of heat removal capability. In the worst case, even flow reversal might happen. Thus at the design stage it is necessary to determine natural frequency for flow oscillations of the system and ensure that possibility of resonance does not exist. This paper outlines a method for determining natural frequency of any fluid system.

\section{$\underline{\text { Analysis }}$}

To develop method of analysis, we followed a stepwise approach. This involved studying the following.

i) Isothermal fluid in an open loop of constant area of cross section and flow being laminar,

ii) Isothermal fluid in an open loop of constant area of cross section flow being laminar or turbulent,

iii) Isothermal fluid in an open loop with step changes in the area of cross section,

iv) Isothermal fluid in an open loop system having parallel paths as well as area changes,

v) Non-isothermal fluid in an open loop system having parallel paths as well as area changes,

vi) Non-isothermal fluid in a closed loop having parallel paths as well as area changes.

The cases (i) to (iv) have been dealt in detail in [1]. We concentrate here on the last two cases since they are of direct practical relevance. 


\section{Variable Temperature Systems}

The behaviour of an open loop system (Fig. 2) can be described by the generalised conservation equation of energy. The energy balance equation for such a system is [4],

$$
\frac{d}{d t}(U+K+\Phi)=\Delta\left[\left(H+\frac{1}{2} \frac{v^{3}}{v}+\phi\right) w\right]-E_{v}-W .
$$

In a practical residual heat removal system, temperature distribution and flow rates are interlinked. Fluid oscillations would also influence heat transfer. However, to simplify the analysis, this influence has been neglected. The temperature distribution of the fluid in the tube is assumed to be known. In addition, any change in fluid temperature due to viscous dissipation is neglected. Because of this, the momentum balance equation and the energy balance equations are treated separately. Due to these assumptions, change in $U$ is neglected. Since there is no flow to or from the system, $w=0$. The individual terms of the equation (1) are derived hereafter.

\section{Kinetic Energy Term}

The first term on the Left-hand side is,

$$
\frac{d}{d t} K=\frac{d}{d t}\left(\int \frac{1}{2} \rho v^{2} d V\right)=\frac{d}{d t} \int_{0}^{L_{T}} \int_{0}^{R} \frac{1}{2} \rho v^{2} 2 \pi r d r d l=\frac{d}{d t} \sum_{i} \frac{1}{2} \pi R_{i}^{2} \int_{0}^{L_{i}} \rho_{i} v_{i}^{2} d l \text {. }
$$

Here the velocity is position dependent due to variation of temperature along the length of the pipe. From continuity it follows that,

$$
\rho_{1} v_{1} A_{1}=\rho_{2} v_{2} A_{2}=\rho_{a v} v_{a v} A \text {. }
$$

Hence the kinetic energy term is,

$$
\frac{d}{d t} K=\frac{1}{2} \frac{d}{d t} \sum_{i} \pi R_{i}{ }^{2} \int_{0}^{L_{i}} \rho\left(\frac{\rho_{a v, i} v_{a v}, i}{\rho}\right)^{2} d l=\frac{1}{2} \sum_{i} \pi R_{i}^{2} \rho_{a v, i}^{2}\left(\int_{0}^{L_{i}} \frac{1}{\rho} d l\right)\left(\frac{d}{d t} v^{2}{ }_{a v}, i\right) .
$$

\section{Potential Energy Term}

Since the system is non-isothermal, the steady state heights would be different. Let the steady state heights of the liquid columns be $\mathrm{K}_{\mathrm{a}}$ and $\mathrm{K}_{\mathrm{b}}$. When the column is set into motion, at any instant of time, let $\mathrm{H}_{\mathrm{a}}$ and $\mathrm{H}_{b}$ be heights of the liquid columns (Fig. 2). The potential energy term can then be calculated as, 


$$
\begin{aligned}
\frac{d}{d t} \Phi & =\frac{d}{d t} \iint_{0}^{L R} \rho g h 2 \pi r d r d h=g \frac{d}{d t}\left(\int_{0}^{H_{a}} \pi r^{2} \rho h d h+\int_{0}^{H_{b}} \pi r^{2} \rho h d h\right) \\
& =g \frac{d}{d t}\left(\int_{0}^{K_{a}} \pi r^{2} \rho h d h+\int_{K_{a}}^{K_{a}+h_{a}} \pi r^{2} \rho h d h+\int_{0}^{K_{b}} \pi r^{2} \rho h d h+\int_{K_{b}}^{K_{b}} \pi r^{2} \rho h d h\right)
\end{aligned}
$$

Here it is assumed that $\mathrm{H}_{a}+\mathrm{H}_{b}=\mathrm{L}$ and the amplitude of oscillation is much smaller than the length of the pipe sections. Since derivative of the first and the third term with respect to time is equal to zero,

$$
\begin{aligned}
\frac{d}{d t} \Phi & =g \frac{d}{d t}\left[\pi R_{a}{ }^{2} \rho_{a}\left[\frac{h^{2}}{2}\right]_{K_{a}}^{K_{a}+h_{a}}+\pi R_{b}{ }^{2} \rho_{b}\left[\frac{h^{2}}{2}\right]_{K_{b}}^{K_{b}-h_{b}}\right] \\
& =\frac{g}{2} \frac{d}{d t}\left[\pi R_{a}{ }^{2} \rho_{a}\left(\left(K_{a}+h_{a}\right)^{2}-K_{a}{ }^{2}\right)+\pi R_{b}{ }^{2} \rho_{b}\left(\left(K_{b}-h_{b}\right)^{2}-K_{b}{ }^{2}\right)\right] \\
& =\pi g\left(R_{a}{ }^{2} \rho_{a} h_{a} \frac{d h_{a}}{d t}+R_{b}{ }^{2} \rho_{b} h_{b} \frac{d h_{b}}{d t}-R_{b}{ }^{2} \rho_{b} K_{b} \frac{d h_{b}}{d t}+R_{a}{ }^{2} \rho_{a} K_{a} \frac{d h_{a}}{d t}\right)
\end{aligned}
$$

\section{Viscous Dissipation Term}

The dissipation due to pipe friction and pipe fittings is calculated by [4],

$$
E_{v}=\sum_{i} \frac{1}{2} v_{a v, i}^{2} \frac{L_{i}}{R_{h}} f_{i}+\sum_{i} \frac{1}{2} v_{a v, i}^{2} e_{v, i} .
$$

where, $f_{i}$ is the fanning friction factor and $e_{v, i}$ is the loss coefficient of the pipe section.

\section{Work Term}

Work done by the fluid against external pressure is given by,

$$
W=\pi R_{b}{ }^{2} v_{b} p_{b}-\pi R_{a}{ }^{2} v_{a} p_{a} .
$$

Here $v_{a}$ and $v_{b}$ are the velocities and $p_{a}$ and $p_{b}$ are the pressure exerted at the end of the tube. These values are indicated in Fig. 2.

\section{The Overall Equation}

Using the equations (2) through (5), the overall equation (1) can be written as, 


$$
\begin{gathered}
\frac{1}{2} \sum_{i} A_{i} \rho_{a v, i}^{2}\left(\int_{0}^{L_{i}} \frac{1}{\rho} d l\right) \frac{d}{d t} v_{a v, i}^{2}+\frac{1}{2} \sum_{i}\left(\frac{L_{i} f_{i}}{R_{h, i}}+e_{v, i}\right) v_{a v, i} \\
+g\left(A_{a} \rho_{a} h_{a} \frac{d h_{a}}{d t}+A_{b} \rho_{b} h_{b} \frac{d h_{b}}{d t}-A_{b} \rho_{b} K_{b} \frac{d h_{b}}{d t}+A_{a} \rho_{a} K_{a} \frac{d h_{a}}{d t}\right) \\
=A_{b} v_{b} p_{b}-A_{a} v_{a} p_{a} .
\end{gathered}
$$

In any pipe section, the velocity $v_{i}=\frac{d h_{i}}{d t}$. Also, $h_{1} A_{1}=h_{2} A_{2}=h_{i} A_{i}=h A_{r}$, where $A_{r}$ is a reference area. Then,

$$
\frac{d h_{1}}{d t}=\frac{A_{r}}{A_{1}} \frac{d h}{d t} \quad \text { or, } v_{1}=\frac{A_{r}}{A_{1}} v \quad \text { and } \quad \frac{d h_{2}}{d t}=\frac{A_{r}}{A_{2}} \frac{d h}{d t} \quad \text { or, } v_{2}=\frac{A_{r}}{A_{2}} v,
$$

in general, $\quad \frac{d h_{i}}{d t}=\frac{A_{r}}{A_{i}} \frac{d h}{d t} \quad$ or, $v_{i}=\frac{A_{r}}{A_{i}} v$.

Now the equation (6) can be written as,

$$
\begin{aligned}
& \sum_{i} A_{i} \rho_{a v, i}^{2}\left(\int_{0}^{L_{i}} \frac{1}{\rho} d l\right) v_{a v, i} \frac{d}{d t} v_{a v, i}+\frac{1}{2} \sum_{i}\left(\frac{L_{i} f_{i}}{R_{h, i}}+e_{v, i}\right) v_{a v, i}^{2} \\
& \quad+g\left(A_{a} \rho_{a} h_{a} v_{a}+A_{b} \rho_{b} h_{b} v_{b}-A_{b} \rho_{b} K_{b} v_{b}+A_{a} \rho_{a} K_{a v_{a}}\right)=A_{b} v_{b} p_{b}-A_{a} v_{a} p_{a} .
\end{aligned}
$$

Expressing velocities in terms of velocity based on the reference diameter,

$$
\begin{gathered}
\sum_{i} A_{i v} \rho_{a v, i}^{2}\left(\int_{0}^{L} \frac{1}{\rho} d l\right) \frac{A_{r}}{A_{i}} v_{r} \frac{d}{d t}\left(\frac{A_{r}}{A_{i}} v_{r}\right)+\frac{1}{2} \sum_{i}\left(\frac{L_{i} f_{i}}{R_{h, i}}+e_{v, i}\right)\left(\frac{A_{r}}{A_{i}} v_{r}\right)^{2} \\
+g\left(A_{a} \rho_{a} h_{a} \frac{A_{r}}{A_{a}} v_{r}+A_{b} \rho_{b} h_{b} \frac{A_{r}}{A_{b}} v_{r}-A_{b} \rho_{b} K_{b} \frac{A_{r}}{A_{b}} v_{r}+A_{a} \rho_{a} K_{a} \frac{A_{r}}{A_{a}} v_{r}\right) \\
=A_{b} p_{b} \frac{A_{r}}{A_{b}} v_{r}-A_{a} p_{a} \frac{A_{r}}{A_{a}} v_{r} .
\end{gathered}
$$

After simplifying and re-arranging we get,

$$
\begin{gathered}
\sum_{i} \frac{A_{r}}{A_{i}} \rho_{a v, i}^{2}\left(\int_{0}^{L} \frac{1}{\rho} d l\right) \frac{d}{d t}\left(\frac{d h}{d t}\right)+\frac{1}{2} \sum_{i} \frac{A_{r}}{A_{i}^{2}}\left(\frac{L_{i} f_{i}}{R_{h, i}}+e_{v, i}\right) \frac{d h}{d t}+g A_{r}\left[\frac{\rho_{a}}{A_{a}}+\frac{\rho_{b}}{A_{b}}\right] h \\
=\left(\rho_{b} K_{b}-\rho_{a} K_{a}\right)+p_{b}-p_{a}
\end{gathered}
$$

Let

$$
T_{1}=\sum_{i} \frac{A_{r}}{A_{i}} \rho_{a v, i}^{2}\left(\int_{0}^{L} \frac{1}{\rho} d l\right) ; \quad T_{2}=\frac{1}{2} \sum_{i} \frac{A_{r}}{A_{i}{ }^{2}}\left(\frac{L_{i} f_{i}}{R_{h, i}}+e_{v, i}\right)
$$




$$
T_{3}=g A_{r}\left[\frac{\rho_{a}}{A_{a}}+\frac{\rho_{b}}{A_{b}}\right] ; \quad T_{4}=g\left(K_{b} \rho_{b}-K_{a} \rho_{a}\right)+\left(p_{b}-p_{a}\right) .
$$

Hence the equation (7) takes the form,

$$
T_{1} \frac{d^{2} h}{d t^{2}}+T_{2} \frac{d h}{d t}+T_{3} h=T_{4}
$$

This equation is solved by Runge-Kutta $4^{\text {th }}$ order method [5].

Initial level of liquid in the limbs will be different due to difference in temperature of the liquid along the length. The level of liquid in both the limbs are estimated and are used in calculations. Three types of initial conditions are possible, viz.,

(i) at $\mathrm{t}=0, h=h_{0}$ and $\frac{d h}{d t}=0 \quad p_{a}=p_{b}$ for all values of $\mathrm{t}$,

(ii) at $\mathrm{t}=0, h=0$ and $\frac{d h}{d t}=0 p_{a} \neq p_{b}$ for all values of $\mathrm{t}$,

(iii) or a combination of both of the above.

Results for these initial conditions are analysed and given hereafter.

TABLE 1

\begin{tabular}{|c|c|c|c|c|c|}
\hline $\begin{array}{l}\text { Pipe } \\
\text { No. }\end{array}$ & Location & Temperature & $\begin{array}{l}\text { Pipe } \\
\text { No. }\end{array}$ & Location & Temperature \\
\hline \multirow{6}{*}{1} & 0.00 & 20.0 & \multirow{2}{*}{3} & 0.80 & 40.0 \\
\hline & 0.20 & 30.0 & & 1.00 & 35.0 \\
\hline & 0.30 & 33.0 & \multirow{6}{*}{4} & 0.00 & 35.0 \\
\hline & 0.50 & 35.0 & & 0.10 & 32.0 \\
\hline & 0.80 & 32.0 & & 0.30 & 38.0 \\
\hline & 1.00 & 30.0 & & 0.40 & 35.0 \\
\hline \multirow{5}{*}{2} & 0.00 & 30.0 & & 0.75 & 38.0 \\
\hline & 0.30 & 32.0 & & 1.00 & 40.0 \\
\hline & 0.50 & 38.0 & \multirow{6}{*}{5} & 0.00 & 40.0 \\
\hline & 0.75 & 40.0 & & 0.20 & 35.0 \\
\hline & 1.00 & 45.0 & & 0.40 & 30.0 \\
\hline \multirow{3}{*}{3} & 0.00 & 45.0 & & 0.50 & 25.0 \\
\hline & 0.30 & 49.0 & & 0.80 & 20.0 \\
\hline & 0.70 & 45.0 & & 1.00 & 20.0 \\
\hline
\end{tabular}

Temperature Distribution of the Open Loop Variable Temperature System

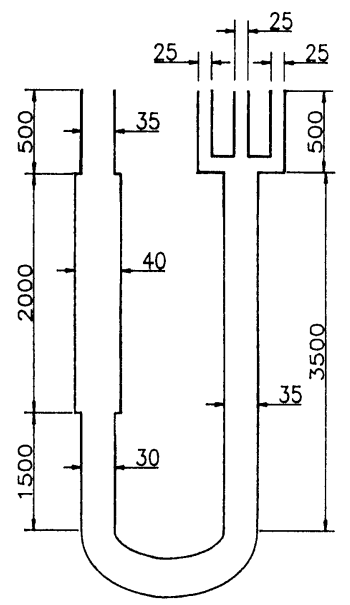

FIG.3

Variable area variable temperature system 
It is assumed that due to an external perturbation, some difference between the steady state levels of the liquid column is created and then it is set free to oscillate. The geometry of the piping system is given in the Fig 3. The temperature distribution of the fluid in the pipes used is given in the Table 1. This distribution is one of the many used for analysis.

With these conditions, the system was analysed. Fig. 4 gives the change in the height of the liquid column with respect to the initial height for left column (column ' $a$ '). For column 'b', results differ only slightly and on the plot they will appear the same and so have not been plotted.

In the second case, an external pressure is applied $(a t t=0)$ to one of the limbs of the system in steady state and is maintained thereafter. On application of the differential pressure, the fluid starts oscillating and attains a new steady state. This behaviour is depicted in the Fig. 5.

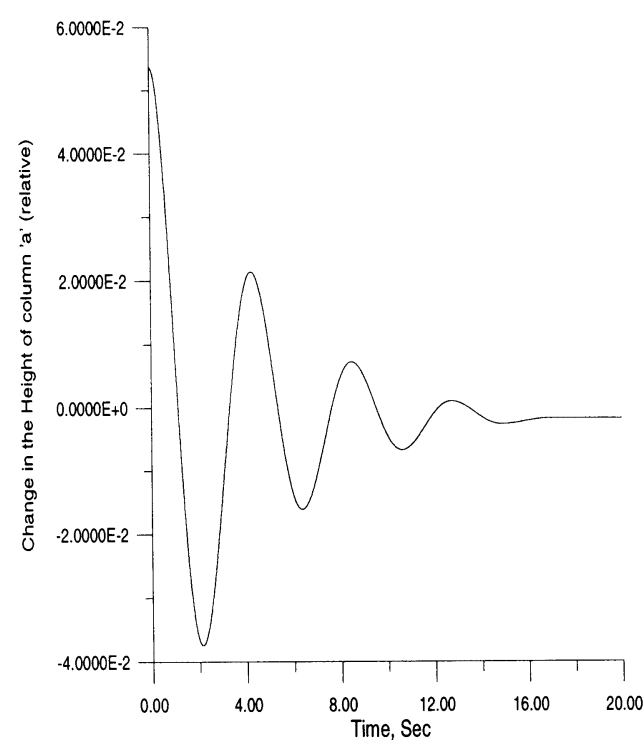

FIG. 4

Results for the case, where initial height of the limbs are different

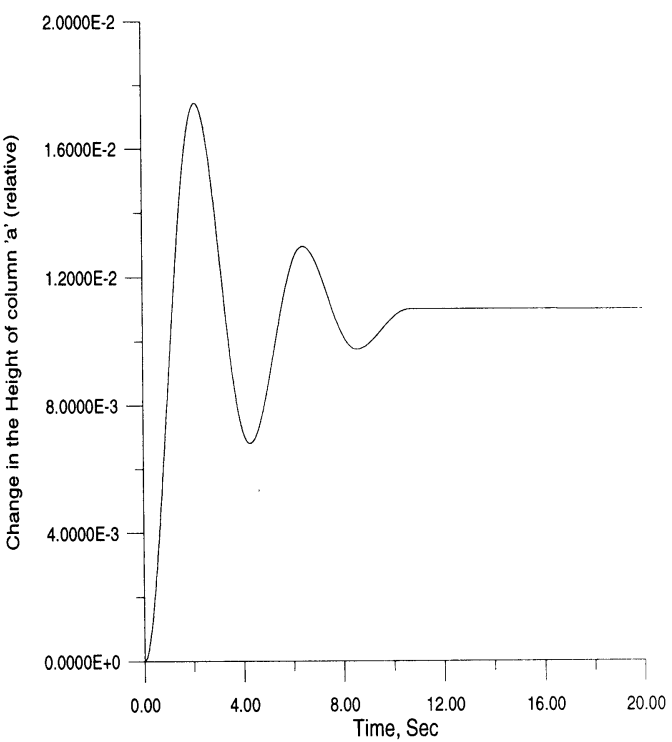

FIG. 5

Results for the case, where initial pressure on the limbs are different

Thus, the response for the case, when the temperature distribution of the fluid is specified is computed. Here temperature distribution was assumed. In order to analyse the response of the RHRS when subjected to oscillations, the temperature and quality distribution of the working fluid in the loop has to be calculated. The method of this calculation has been reported earlier $[8,9]$. 


\section{Closed Loop Systems}

A simplified scheme of the second loop of the natural circulation residual heat removal system is indicated in the Fig 6.

The mathematical model developed in previous section deals with an open loop, variable temperature system. The RHR system is a closed loop. The boiling region and the superheated region in the heat exchanger, the steam pipes and the condensing region of the condenser are also to be included for analysing the Natural Circulation System.

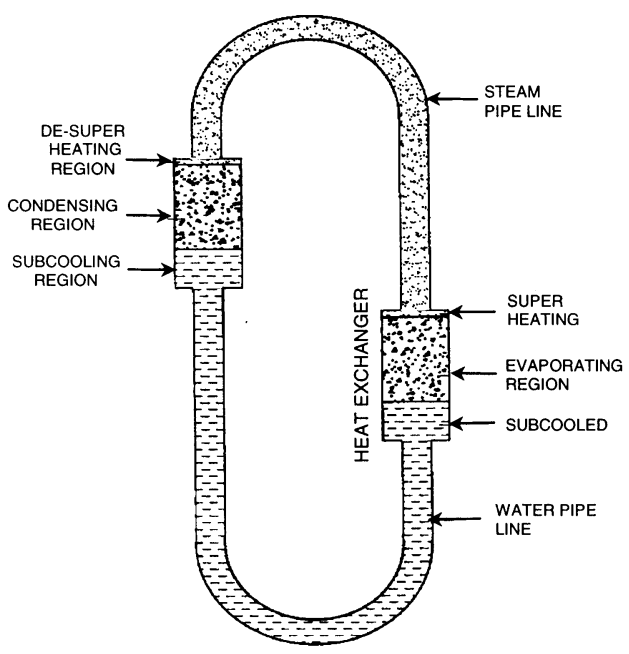

FIG. 6

Schematic of closed loop two phase residual heat removal system

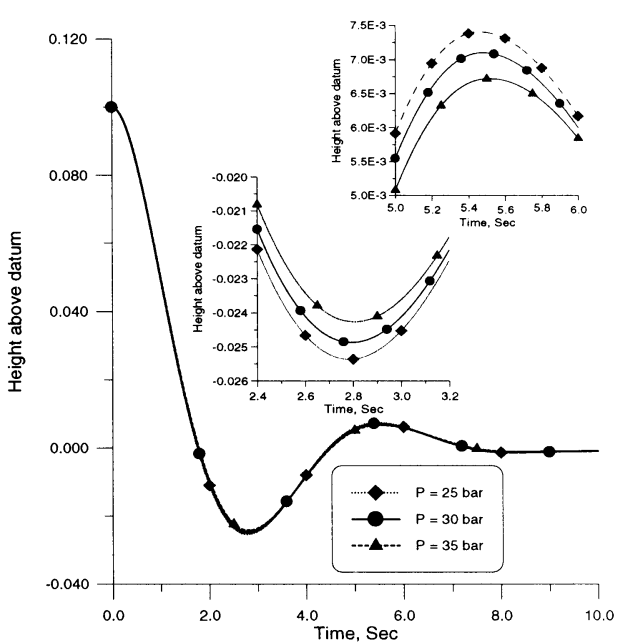

FIG. 7

Response of the RHR system

In the previous section, the density and viscosity of the working fluid were evaluated as functions of temperature. However in the present case, as two-phase mixture is involved in the heat exchanger and in the condenser, the density and viscosity are to be evaluated as a function of temperature and or quality as appropriate. In the open loop system considered in section 2.1, the external pressure acting on one of its limbs can be different from the external pressure acting on the other limb. In the present case, where a closed loop is being considered, movements of the liquid meniscuses are in phase and the change in the volume of the fluid due to oscillations is very small as compared to the total volume of the system [1]. Hence, in the closed loop system, the pressure acting on both the liquid meniscuses will be the same and it is assumed to remain constant. To maintain the flow rate through the loop, the liquid in the condenser is 
at a higher elevation than that in the heat exchanger. The difference in the levels is calculated by equating the head generated in the two limbs as a result of difference in density. The work term in the present case turns out to be zero.

Viscous dissipation term $E_{v}$ includes resistance to motion offered by the steam path and both the pipe friction and fitting losses are accounted for. The pressure drop in the pre-heating and superheating regions of the heat exchanger, sub-cooling region of the condenser and in the steam and water pipe lines are calculated [10]. Pressure drops in the boiling region of the heat exchanger and the condensing region of the condenser are evaluated using the Martinelli-Nelson method [6].

To evaluate the kinetic energy term, integration of specific volume of the working fluid over the entire loop is to be carried out. The ESSAP program [9] generates the temperature and quality distribution of the working fluid throughout the loop. Using this information, density of the fluid at various locations in the loop is calculated considering both temperature and quality distributions as appropriate. Numerical integration by Simpson's $1 / 3^{\text {rd }}$ rule [5] is employed for each part of the loop to estimate the integral. The results of integration for each of the part is multiplied by $\rho_{r, i}^{2} A_{r} / A_{i}$ and are summed up to get the value of the kinetic energy term.

Following the development in the previous section, the overall governing equation can be written as,

$$
T_{1} \frac{d^{2} h}{d t^{2}}+T_{2} \frac{d h}{d t}+T_{3} h=T_{4} .
$$

The coefficients $T_{1}, T_{2}, T_{3}$ and $T_{4}$ of the equation are given by,

$$
\begin{array}{rlrl}
T_{1} & =\sum_{i} \frac{A_{r}}{A_{i}} \rho_{a v, i}^{2}\left(\int_{0}^{L} \frac{1}{\rho} d l\right) ; & T_{2}=\frac{1}{2} \sum_{i} \frac{A_{r}}{A_{i}^{2}}\left(\frac{L_{i} f_{i}}{R_{h, i}}+e_{v, i}\right) ; \\
T_{3}=g A_{r}\left[\frac{\rho_{a}}{A_{a}}+\frac{\rho_{b}}{A_{b}}\right] ; & T_{4}=g\left(K_{b} \rho_{b}-K_{a} \rho_{a}\right)+\left(p_{b}-p_{a}\right) .
\end{array}
$$

Where the terms are evaluated for the case of two phase closed loop as discussed above.

\section{Program Description}

A program ORBITS (OscillatoRy BehavIour of residual heaT removal System) has been developed for carrying out the analysis of oscillations of the Residual Heat Removal System. The program has an open structure. The components, which constitute the loop, are specified in a modular 
manner. In this case the possible components are; (i) Pipelines (may be water pipeline or steam pipeline), (ii) heat exchanger and (iii) condenser. Different numbers of each of the components can be specified. The temperature and quality of the working fluid at various locations along the length of the pipe sections, the heights of pre-heating, evaporating and superheating regions of heat exchangers, and heights of the condensing and subcooling sections for condenser are inputs. Each of the components is subdivided into a number of segments during the calculations. The thermal and transport properties of water and steam are calculated by using the methodology of the property routine STH2Ox used in RELAP [7] program. The coefficients $T_{1}, T_{2}, T_{3}$ and $T_{4}$ are evaluated. This equation is then solved by Runge-Kutta $4^{\text {th }}$ order method.

\section{$\underline{\text { Results }}$}

The results of oscillatory behaviour of the system for three operating pressures are given in Fig. 7 . The results indicate that the system is highly damped due to the presence of two-phase regions in the heat exchanger and the condenser. When the operating pressure is increased, the length of the boiling region and the condensing region increases. Due to this effect, the time period of oscillations increases. However, the changes are small. Hence, it can be stated that small changes in operating pressure of the system do not affect the frequency of oscillations significantly.

\section{Conclusion}

A program has been developed to compute natural frequency of fluid oscillations in a closed system. For the system studied, the natural period of oscillation is about $5.5 \mathrm{sec}$ and is not significantly influenced by operating pressure. Due to seawater waves, the period of oscillation of a barge (located in the Arabian Sea / Bay of Bengal) on which a Nuclear Desalination Plant of the type described here may be mounted is about 12 to $15 \mathrm{sec}$. Since the natural frequency of oscillation of the system is away from the frequency of oscillations induced by sea waves, the chances of resonant oscillations of the residual heat removal system and its consequential inability to perform the intended function is remote.

\section{Nomenclature}

\begin{tabular}{lll|lll}
$A$ & Area & $\mathrm{m}^{2}$ & $R$ & Radius & $\mathrm{m}$ \\
$C_{p}$ & Specific heat & $\mathrm{J} \mathrm{kg}^{-1} \mathrm{~K}^{-1}$ & $r$ & $\begin{array}{l}\text { distance from the } \\
\text { centre along the radius }\end{array}$ & $\mathrm{m}$
\end{tabular}




\begin{tabular}{lll|lll|ll}
$D$ & Diameter of Tube & $\mathrm{m}$ & $R e$ & Reynolds Number & $-\cdot$ & \\
$E_{v}$ & Viscous dissipation & $\mathrm{J}$ & $t$ & Time & $\mathrm{s}$ & \\
$e_{v}$ & $\begin{array}{l}\text { Total Loss coefficient of } \\
\text { pipe section }\end{array}$ & - & $U$ & Internal Energy & $\mathrm{J}$ & \\
$f$ & fanning friction factor & -- & $v$ & Velocity of fluid & $\mathrm{m} \mathrm{s}^{-1}$ & \multicolumn{2}{|l}{ SUBSCRIPTS } \\
$g$ & Acceleration due to gravity & $\mathrm{m} \mathrm{s}^{-2}$ & $V$ & Volume & $\mathrm{m}^{3}$ & $I$ & location 1 \\
$G$ & mass flux & $\mathrm{kg} \mathrm{m}^{-2} \mathrm{~s}^{-1}$ & $W$ & Work & $\mathrm{J}$ & 2 & location 2 \\
$h$ & height of liquid column & $\mathrm{m}$ & $z$ & distance along the axis & $\mathrm{m}$ & $a$ & Location a \\
$H$ & Enthalpy & $\mathrm{J}$ & $\Phi$ & Potential Energy & $\mathrm{J}$ & $b$ & Location b \\
$h$ & heat transfer coefficient & $\mathrm{W} \mathrm{m} \mathrm{m}^{-2}$ & $\mu$ & Viscosity & $\mathrm{Pa} . \mathrm{s}$ & $a v$ & Average \\
$K$ & Kinetic Energy & $\mathrm{J}$ & $\rho$ & Density & $\mathrm{kg} \mathrm{m}^{-3}$ & $h$ & Hydraulic \\
$k$ & Thermal conductivity & $\mathrm{W} \mathrm{m} \mathrm{K}^{-1}$ & $T$ & Period of oscillation & $\mathrm{s}$ & $i$ & Section i \\
$L$ & Length of Pipe Section & $\mathrm{m}$ & $v$ & Specific volume & $\mathrm{m}^{3} \mathrm{~kg}^{-1}$ & $n$ & natural \\
$p$ & Pressure & $\mathrm{Pa}$ & $\omega$ & frequency of oscillation & $\mathrm{Hz}$ & $T$ & total
\end{tabular}

\section{References}

1. J.S.Jayakumar, "Analysis of Two-phase Natural Circulation System under Oscillatory conditions", MSc(Engg.) thesis, Indian Institute of Science, Bangalore, April 2000.

2. IAEA Code on the "Safety of Nuclear Power Plants: Design", Safety Series No. 50-C-D (Rev-1), 1988.

3. Samailov. O.B., Usinin. G.B., and Bakhmetiev. A.M., "Safety of Nuclear Power Plants", Energoatomizhdat, 1989 (in Russian).

4. Bird R. B., Stewart W.E., Lightfoot E. N., "Transport Phenomena", 1960.

5. Press W. H, Teukolsky S. A, Vetterling W. T., Flannery B. P.,"Numerical Recipes in C”, Cambridge University Press, 1993.

6. Collier John G., "Convective Boiling and Condensation", McGraw Hill, 1972.

7. "A Computer Programme for Transient Thermal Hydraulic Analysis of Nuclear Reactors and Related Systems", ANCR-NUREG-1335, 1976.

8. Ranajit Manna, J.S.Jayakumar and R.B.Grover, "Thermal Hydraulic Design of a Condenser for a Natural Circulation System”, J. Energy, Heat and Mass Transfer, Vol. 18, 1996.

9. J. S. Jayakumar and R. B. Grover, "Two phase Natural Circulation Residual Heat Removal", Proc. $3^{\text {rd }}$ ISHMT-ASME Heat and Mass Transfer Conference, Kanpur, 1997.

10. Idelchik I.E., "Handbook of Hydraulic Resistance”, Hemisphere, 1986.

Received January 29, 2002 